\title{
ECOLOGICAL PERSPECTIVE OF THE DIVERSITY OF FUNCTIONAL RESPONSES
}

\author{
AHMAD PERVEZ ${ }^{1, *}$, PREET PAL SINGH${ }^{2}$, and HAKAN BOZDOĞ HN $^{3}$ \\ ${ }^{1}$ Biocontrol Laboratory, Department of Zoology, Radhey Hari Government P. G. College, Kashipur - 244713, Udham Singh Nagar, \\ Uttarakhand, India \\ 2 Department of Mathematics, Government P. G. College, Rishikesh, Uttarakhand, India \\ ${ }^{3}$ Ahi Evran University, Vocation School of Technical Sciences, Department of Plant and Animal Production, 40100, Kırşehir, Turkey \\ *Corresponding Author: ahmadpervez@yahoo.com
}

\begin{abstract}
Prey-predator interactions have been modelled by numerous workers. Ecologists have continuously modified Lotka-Volterra equations in order to provide more realistic descriptions of the complexity of these interactions. The response of predator(s) to increasing prey density can be best described in terms of a functional response, which is an important criterion determining the success or failure of predator(s) to control fluctuating prey populations. The functional response of a predator is further differentiated into Holling's Type I, II, III, IV and V. We discuss one-prey and one-predator interactions, in which the models are modified by the inclusion of steady-state satiation and growth factors. We review situations where two prey and one predator interact, and vice versa. We also discuss Holling's Type IV model relevant to competition and food chains. There is a need to examine functional responses as these models were mostly developed by pure mathematicians and their relevance to field conditions remains largely untested. Prey-predator interactions can be affected even by small factors and ecologists should include these models in their experimental design when attempting to predict realistic interactions.
\end{abstract}

Keywords: dynamics; ecology; Holling; modelling; predator; prey

\section{Introduction}

Prey-predator interactions stimulated theoretical ecologists to develop equations that described realistic interactions. Lotka (1925) and Volterra (1926) use differential equations to describe these interactions. However, the complexity of prey-predator interactions resulted in subtle modifications in the proposed equations. Nicolson and Bailey (1935) introduced the term "area of discovery" as a variable associated with the attack rate of the predator, which triggered a chain of alterations in Lotka-Volterra models, especially when prey and predator densities are varying. The functional response of a predator describes its rate of prey consumption at different prey densities (Holling 1959, 1965), which can be classified into five types (Type I, II, III, IV and V). Equation $c(x)$ $=m x$ describes the Type I response, in which there is a linear increase in predation rate with prey density up to a threshold (Leslie and Gower 1960; Hsu and Huang 1995). Holling (1959) used Michaelis-Menten's formula (expressed by $c(x)=m /(A+x)$ ) to describe the Type II functional response in which there is a decelerating increase in predation rate with prey density until satiation is attained (Aziz-Aloui and Okiye 2003; Huu Du et al. 2007).

Holling's Type III response exhibits a sigmoidal increase in predation rate at high prey densities and is described by the equation $c(x)=m x^{n} /\left(A+x^{n}\right)$. The general form of this type of functional response was developed by Kazarinov and van den Driessche (1978). Type IV response is relatively less studied and describes the condition where the predator per capita predation rate decreases at exceptionally high prey densities, which is expressed by the equation $c(x)=m x^{2} /(A+x)(B+x)$ (Tanner 1975; Ali et al. 2016a,b). Type V response, also known as Ivlev's functional response, is described by the equation, $c(x)=m\left(1-e^{-A x}\right)$. Holling's Type II and III responses could also be considered as classical Hill functions (Gesztelyi et al. 2012). However, random perturbations in population dynamics can lead to very different models, which are more realistic simulations (Xianning and Lansun 2003; Bing et al. 2004). This diversity and complexity in prey-predator interactions stimulated us to present here an overview of the mathematical models that may better predict such interactions.

\section{Various types of prey-predator systems}

Prey-predator models depend on the nature and quantity of prey and predators. Numerous factors affect predators and their prey. We review a few situations where the predator is expected to behave differently and this change in behaviour possibly affects its functional response.

\section{One Prey - One Predator System}

There are many functional response studies of one prey and one predator (particularly ladybirds, see book: Hodek et al. 2012). Holling's $(1959,1965)$ equations did not include the effect of satiation. However, if needed, it might be included in a steady state satiation (SSS) equation (Jeschke et al. 2002) with a constant satiation rate. Jeschke et al. (2002) suggest that digestion should not be incorporated as part of prey handling time (Pervez and Omkar 2003), as it does not prevent foraging. Instead, Jeschke et al. (2002) incorporate hunger level and time lost in unsuccessful attempts to catch prey in their model and named it as steady-state satiation (SSS) equation. In this equation, attack rate (a) is the product of encounter rate 
$(\boldsymbol{\beta})$ between a foraging predator and prey, the probability that the consumer detects encountered food $(\gamma)$, the probability that the consumer attacks detected food $(\boldsymbol{\delta})$, and attack efficiency $(\xi)$, i.e. the frequency of successful attacks (i.e. $a=\boldsymbol{\beta} \boldsymbol{\gamma} \boldsymbol{\delta} \boldsymbol{\xi})$. Handling time $(b)$ is the ratio of attack time $t_{\text {att }}$ (per food item) and attack efficiency $(\xi)$, added to eating time $t_{\text {eat }}$ (per food item), i.e. $b=t_{\text {att }} / \xi+t_{\text {eat }}$. This SSS equation is:

$$
y(x)=\left\{\begin{array}{cr}
\frac{1+a x(b+c)-\sqrt{1+a x\left(2(b+c)+a x(b-c)^{2}\right)}}{2 a b c x} & a, b, c, x>0 \\
\frac{a x}{1+a b x} & b>0, c=0 \\
\frac{a x}{1+a c x} & b=0, c>0 \\
a x & b=c=0 \\
0 & a=0 \text { or } x=0
\end{array}\right\}
$$

Jeschke and Hohberg (2008) also propose a satiation model that accounts for prey depletion and increasing predator satiation over time.

$$
\begin{gathered}
\frac{d y(t)}{d t}=\frac{h(t) a x(t)}{1+h(t) a b x(t)} \\
x(t)=x(0)-y(t)
\end{gathered}
$$

Here, the first equation reveals that prey consumption rate increases as a function of hunger level $(h)$, attack rate $(a)$, prey abundance $(x)$ and handling time $(b)$.

Liu and Lin (2010) introduce a cross-diffusion parameter in the prey-predator models. They are of the opinion that prey might be able to protect themselves from attacks by predators and hence a resistance parameter should also be incorporated in the model. Recent research also focuses on including the dynamic properties of random perturbations in classical prey-predator models (Liu et al. 2004; Liu and Tan 2007). Randomly perturbed predator-prey models, thereby, have been used by many researchers to describe the efficiency of integrated pest management strategies (Jeschke et al. 2002; Mailleret and Grognard 2009). For managing pest populations specific prey-predator combinations along with pesticide sprays can also work. These models may further be modified by manipulating the predator's stage structure (Georgescu and Zhang 2010), state-dependent random perturbations, age and defence mechanisms of the pests (Zhang and Georgescu 2010), and patch structure of the pests (Yang and Tang 2009). Thus, it is possible to improve the classical models designed for one-prey and one-predator system.

\section{Incorporation of a Growth Factor in the equation}

Both prey and predator grow logistically in the field and in certain artificial experimental arenas, as the predator has other food sources besides prey. Thus, the predator has two growth rates, viz. predation and logistic growth. Rayungsari et al. (2014) introduce a model that has three equilibrium points, viz. the prey extinction point $\left(\mathrm{E}_{1}\right)$, predator extinction point $\left(\mathrm{E}_{2}\right)$ and survival point $\left(E_{2}\right)$. Here, $E_{2}$ is unstable, while the other two are locally asymptotically stable under certain conditions. Sharma and Samanta (2014) describe a similar model and considered two situations, i.e. normal and hunger. In this model, fractional derivatives of order $\boldsymbol{\alpha}$ and $\boldsymbol{\beta}$ (where $0<\boldsymbol{\alpha} \leq 1 ; 0<\boldsymbol{\beta} \leq 1$ ) are considered rather than only the first order time derivatives. In addition, they construct approximate solutions using the Homotopy Perturbation Method and Variation Iteration Method. The modified equations for the above two cases are presented below.

Case 1: For the normal situation, i.e. the functional response is equal to the attack capacity per predator; (when $\mathrm{cN} \geq \mathrm{aP}$ ):

$$
D_{t}^{\alpha} N(t)=p\left\{\frac{r}{\lambda}\left[1-\left[\frac{N(t)}{k}\right]^{\lambda}\right] N(t)-a P(t)\right\}
$$$$
D_{t}^{\beta} P(t)=p(b-d) P(t)
$$

Case 2: For the hunger situation, the functional response is smaller than the attack capacity, i.e., if more prey were available, the predator would increase its catch (when $\mathrm{cN} \leq \mathrm{aP}$ ):

$$
\begin{gathered}
D_{t}^{\alpha} N(t)=p\left\{\frac{r}{\lambda}\left[1-\left[\frac{N(t)}{k}\right]^{\lambda}-c\right] N(t)\right\} \\
D_{t}^{\beta} P(t)=p\left[b \frac{c}{a} N(t)-P(t)\left[d+\frac{1}{T} \ln \left(\frac{a P(t)}{c N(t)}\right)\right]\right]
\end{gathered}
$$

With initial conditions

$$
\begin{gathered}
N(0)=c_{1} ; P(0)=c_{2} \\
\text { and } 0<\boldsymbol{\alpha} \leq 1 ; 0<\boldsymbol{\beta} \leq 1
\end{gathered}
$$

Here, $N(t)$ and $P(t)$ denote the population density of prey and predator respectively, whereas the other parameters are $r$ (intrinsic growth rate of prey population), $k$ (carrying capacity of prey population), $a$ (attack capacity of predator population), $b$ (birth rate of predator), $c$ (maximum predation rate), $d$ (natural death rate of predator population) and $T$ (the typical time of the response to hunger).

\section{Two Prey - One Predator System}

It is possible to describe the functional response at multiple tritrophic levels, i.e. ecosystems with many species of prey (Georgescu 2011), predators (Pei et al. 2005) and food chains (Baek 2008). Krivan and Eisner's (2006) two-prey and one-predator model predicts that when prey resources grow exponentially, the prey handling times decrease to almost zero and can result in great differences in prey consumption. This could lead to the extinction of weaker prey. In a different two-prey and one-predator model, the prey handling time depends on comparative prey density that enables the predator to stabilize the system (Green 2004). Here, the prey do not compete and predation follows the density gradient of prey. Kesh et al. (2000) propose a two- prey and one-predator species 
model in which the predator dietary functions are ratio-dependent. They predict the conditions that result in the permanent stability of the system. Pal and Mahapatra (2014) introduce an imprecise two prey - one predator model by using interval and fuzzy numbers in the modelling of the prey and predator interaction.

In another two-prey and one-predator model, the intrinsic growth rate of the prey varies periodically in order to incorporate seasonal variations in which the environmental conditions follow a predictable pattern (Song and Xiang 2006). In a similar model using a Lyapunov function, the prey consumption of a single predator increases linearly with increase in density of two species of prey (Chaudhuri and Kar 2004). Thus, it is inevitable that as ecological conditions vary, functional responses have to change and other variables and constants must be incorporated in order to predict more realistic outcomes of prey-predator interactions.

\section{One-Prey - Two-Predator System}

In one-prey and two-predator systems, the two predators can differ in strength. Hsu (1981) proposes one such model and infers that the top predator is more successful if the interference coefficient is small, while the outcome of predation depends on the initial abundances of the two predators if the interference coefficient is large. However, the criterion for persistence and stability in such systems was proposed later by Freedman and Waltman (1984) and Gopalsamy (1986). The fate of the two predators attacking a single prey and their co-existence depends on the ratio between their numerical responses (Mitra et al. 1992). Dubey and Das (2000) propose a model for two predators foraging for a single species of prey based on a Gause-type model. In this, the predators compete with one another when prey are scarce. Dubey and Upadhyay (2004) propose another model for one prey and two predator interactions and discuss the factors resulting in either persistence or extinction. Alebraheem's (2012) model indicates that coexistence of two predators ( $y$ and $z$ ) depends on the efficiencies of the predators' conversion of prey biomass into predator offspring being similar. However, if they differ the predator with the lower value becomes extinct. Sunaryo et al. (2013) propose a similar model in which both the top and second predator have Type III functional responses. This model predicts two equilibrium points and a switch from instability to stability known as Hopf bifurcation. Garay et al. (2015) suggest that in two-predator systems there will be a higher incidence of fighting and lower incidence of killing prey. Experimental studies reveal that in such scenarios the two predators can work in tandem by complimenting each other. Laboratory experiments indicate a synergistic effect on prey mortality when two ladybird-predators (Coleoptera: Coccinellidae) attack the same population of aphids (Homoptera: Aphididae) (Omkar and Pervez 2004, 2011). The mortality of pea aphids (Acyrthosiphon pisum) infesting a crop of alfalfa
(Medicago sativa) increases many-fold when attacked by three natural enemies, viz. a ladybird beetle Harmonia axyridis (Pallas), damsel bug Nabis sp. and parasitic wasp Aphidius ervi (Cardinale et al. 2003).

\section{Holling Type IV Model for Describing Competition / Food Chains}

Andrews (1968) suggests a function known as the Monod-Haldane function (i.e. $p(x)=m x /\left(a+b x+x^{2}\right)$, which is also known as Holling's Type IV function with a non-monotonic equation. It may also be written as: $g(N, P)=a N /\left(1+b N+a N^{2}\right)$. This equation was further simplified by Sokol and Howell (1987) who assume that $b=0$, which nullifies the $b N$ part of the denominator.

Shuwen et al. (2005) investigate two-species predatorprey systems using Holling's Type IV response with predator subject to random perturbations. Funasaki and Kot (1993) report chaos in a periodically pulsed mass-action chemostat. Venkatesan et al. (2003) discuss the presence of a multiple period-doubling bifurcation route to chaos in periodically pulsed chaotic dynamic systems. Baek et al. (2009) describe conditions for the stability of a food chain using Holling type IV functional response and the Floquet theory of impulsive equations and small perturbations. Upadhyay et al. (2011) studied a model system subject to environmental driving forces that are unable to drive the system from a regime of deterministic chaos towards a stochastically stable situation. Upadhyay and Raw (2011) study the complex dynamics of a three-species food chain using a Holling Type IV functional response and report chaotic behaviour in a narrow region of the bifurcation parameter space for biologically realistic parameter values. Ali et al. (2016b) infer that increasing or decreasing the top predator's intrinsic growth rate can change the dynamics of food chains from chaotic to asymptotically stable. In addition, an increase in the death rate of the middle predator can stabilize the dynamics of this system.

Some predator-prey systems include two predator species attacking a single species of prey. Of the two predators, the top predator attacks both the prey and the second predator when the prey is scarce. Different approaches simulate the complex interactions of this multi-predator system with prey density or extra guild prey, which are complicated, multi-variant and chaotic (Funasaki and Kot 1993; Venkatesan et al. 2003; Shuwen et al. 2005). In such circumstances or in food chains, complex simultaneous functional responses occur. A depletion in prey density affects the attack rate of the top predator (X) and second predator $(\mathrm{Y})$. Simultaneously, the density of Y will also decrease because of X. Zhang and Chen (2005) present a modified model that may depict the outcome of these complex interactions.

Cosner and de Angelis (1999) introduce the concept of the effect of spatial grouping on the functional response of predators. They state that if predators have a homogenous spatial distribution, then a prey depend- 
ent functional response can be expected. However, when shoals of predators hunt for prey, the functional response will be both predator and prey dependent. This results in the predator density having a significant effect on the functional response, which is then dependent on predator density and the encounter rate. Many predators become confused on encountering a swarm of prey, which negatively affects their predation rate (Jeschke and Tollrian 2005). At high prey densities, it may restrict the predator's neuronal abilities, which makes them less successful in attacking prey (Krause and Ruxton 2002). Predator confusion significantly affects the functional response, however, this effect is not included in traditional models (Jeschke and Tollrian 2005). Most of these models only describe the ecological interaction between predator and prey. In predator confusion situations, usually the functional response is dome-shaped, indicating a dramatic dip in the predation rate at high densities. Predator confusion is widespread and occurs in many animal systems. Interestingly, predator-prey theory, especially functional response models, do not include it as a factor reducing predation rate. This is addressed by Jeschke and Tollrian (2005) in their elaborate model based on the SSS equation proposed by Jeschke et al. (2002). Olson et al. (2013) indicate that swarming evolves as an emergent behaviour in prey when a simple perceptual constraint, predator confusion, is imposed on the predator. In addition, measuring swarm density and dispersion serves as a useful alternative for qualitative assessment of every swarm (Huepe and Aldana 2011).

\section{Conclusion}

It is evident from this overview that functional responses are highly sensitive to biological and ecological changes. We describe a couple of models in detail just to emphasize the importance of variables that are often neglected by biologists in functional response studies. A subtle change in ecological conditions directly or indirectly affects the possible outcome of prey-predator interactions. Hence, models that incorporate all the major and minor factors that may affect them should be developed. Although there is a plethora of mathematical models, which include many of the factors that affect functional response, they are rarely used or tested by ecologists. Hence, there is an urgent need to test these models using both laboratory and field studies.

\section{Acknowledgements}

Authors thank to Dr. Barbara Castleton, ESL Instructor, South Seattle College, Seattle, Washington, USA for improving the English of this manuscript. AP thanks SERB, Department of Science and Technology, New Delhi for financial assistance (EMR/2016/006296).

\section{REFERENCES}

Alebraheem J (2012) Persistence of predators in a two predators-one prey model with non-periodic solution. Appl Math Sci 6: 943-956.

Ali SJ, Arifin NM, Naji RK, Ismail F, Bachok N (2016a) Dynamics of Leslie-Gower model with simplified Holling Type IV functional response. J Nonlin Sys Appl 5: 25-33.

Ali SJ, Arifin NM, Naji RK, Ismail F, Bachok N (2016b) Boundedness and Stability of Leslie-Gower Model with Sokol-Howell Functional Response. In: Kiliçman A et al. (eds) Recent Adv Math Sci, pp 13-26.

Andrews JF (1968) A mathematical model for the continuous culture of macroorganisms utilizing inhibitory substrates. Biotech Bioeng 10: 707-723.

Aziz-Aloui MA, Okiye MD (2003) Boundedness and Global Stability for a Predator-Prey Model with Modified LeslieGower and Holling-Type II Schemes. Appl Math Letters 16: 1069-1075.

Baek H (2008) Dynamic complexities of a three-species Beddington-DeAngelis system with impulsive control strategy. Acta Applicandae Mathematicae 110: 23-38.

Baek H, Do Y, Saito Y (2009) Analysis of an impulsive predator-prey system with Monod-Haldane functional response and seasonal effects. Math Prob Eng, pp 1-16.

Bing L, Yujina Z, Lansun C (2004) Dynamic complexities of a Holling I predator-prey model concerning biological and chemical control. Chaos, Solitons and Fractals 22: 123-34.

Cardinale BJ, Harvey CT, Gross K, Ives AR (2003) Biodiversity and biocontrol: Emergent impacts of a multi-enemy assemblage on pest suppression and crop yield in an agroecosystems. Ecol Lett 6: 857-865.

Chaudhuri KS, Kar TK (2004). Harvesting in a two prey - one predator fishery: a Bio economic-model. Anziam J 45: 443-456.

Cosner C, de Angelis DL (1999) Effects of Spatial Grouping on the Functional Response of Predators. Theor Pop Biol 56: 65-75.

Dubey B, Das B (2000) Modelling the interaction of two predators competing for a prey in a diffusive system. Ind J Pure Appl Math 31: 823-837.

Dubey B, Upadhyay RK (2004) Persistence and extinction of oneprey and two-predator system. Nonlinear Analysis: Modelling and Control 9: 307-329.

Freedman HI, Waltman P (1984) Persistence in models of three interacting predator prey populations. Math Biosci 68: 213-231.

Funasaki E, Kot M (1993) Invasion and Chaos in a Periodically Pulsed Mass-Action Chemostat. Theor Pop Biol 44: 203-224.

Garay J, Varga Z, Gámez M, Cabello T (2015) Functional response and population dynamics for fighting predator, based on activity distribution. J Theor Biol 368: 74-82.

Georgescu P (2011) On the impulsive control of a n-prey and one-predator food web model: In: Dumitriu NA, Georgescu P, Strugariu R (eds), Proc Int Conf Appl Pure Math, Iasi, Romania, Bull Inst Pol Iasi LVII(LXI): 111-123.

Georgescu P, Zhang H (2010) An impulsively controlled predator-pest model with disease in the pest. Nonlin Anal Real World Appl 11: 270-287.

Gesztelyi R, Zsuga J, Kemeny-Beke A, Varga B, Juhasz B, Tosaki A (2012) The Hill equation and the origin of quantitative pharmacology. Arch Hist Exact Sci 66: 427-438.

Gopalsamy K (1986) Convergence in resource based competition system. Bull Math Biol 48: 681-699.

Green E (2004) The effect of a 'smart' predator in a one predator, two prey system. https://www.rose-hulman.edumathjournal /archives/2004/vol5-n2/paper5/v5n2-5pd.pdf. 
Hodek I, van Emden HF, Honek I (2012) Ecology and behavior of the ladybird beetles (Coccinellidae). Wiley-Blackwell, Oxford, UK.

Holling CS (1959) Some characteristics of simple types of predation and parasitism. Can Ent 91: 385-398.

Holling CS (1965) Functional response of predators to prey density and its role in mimicry and population regulation. Mem Ent Soc Canada 45: 3-60.

Hsu SB (1981) On a resource based ecological competition model with interference. J Math Biol 12: 45-52.

Hsu SB, Huang TW (1995) Global stability for a class of predator-prey systems. Siam J Appl Math 55: 763-783.

Huepe C, Aldana M (2011) New tools for characterizing swarming systems: A comparison of minimal models. Physica A 387: 2809-2822.

Huu Du N, Man NM, Trung TT (2007) Dynamics of predator-prey population with modified Leslie-Gower and Holling-type II schemes. Acta Math Vietnam 32: 99-111.

Jeschke JM, Hohberg K (2008) Predicting and testing functional responses: An example from a tardigrade-nematode system. Basic Appl Ecol 9: 145-151.

Jeschke JM, Tollrian R (2005) Effects of predator confusion on functional responses. Oikos 111: 547-555.

Jeschke JM, Kopp M, Tollrian R (2002) Predator functional responses: discriminating between handling and digesting prey. Ecol Monogr 72: 95-112.

Kazarinov N, van den Driessche P (1978) A model predator-prey systems with functional response. Math Biosci 39: 125-134.

Kesh D, Sarkar AK, Roy AB (2000) Persistence of two prey - one predator system with ratio dependent predator influence. Math Meth Appl Sci 23: 347-356.

Krause J, Ruxton GD (2002) Living in Groups. Oxford University Press, Oxford.

Krivan V, Eisner J (2006) The effect of Holling Type II functional response on apparent competition. Theor Pop Biol 70: 421-430.

Leslie PH, Gower JC (1960) The properties of a stochastic model for the predator-prey type of interaction between two species. Biometrika 47: 219-234.

Liu J, Lin Z (2010) Stationary patterns for a predator-prey model with Holling type III response function and cross-diffusion. Bull Korean Math Soc 47: 251-261.

Liu B, Zhi Y, Chen L (2004) The dynamics of a predator-prey model with Ivlev's functional response concerning integrated pest management. Acta Math Appl Sin Engl Ser 20: 133-146.

Liu Z, Tan R (2007) Impulsive harvesting and stocking in a Monod-Haldane functional response predator-prey system. Chaos, Solitons and Fractals 34: 454-464.

Lotka AJ (1925) The Elements of Physical Biology, Williams and Wilkins, Baltimore.

Mailleret L, Grognard F (2009) Global stability and optimisation of a general impulsive biological control model. Math Biosci 221: 91-100.

Mitra D, Mukherjee D, Roy AB (1992) Permanent coexistence in a resource-based competition system. Ecol Model 60: 77-85.

Nicholson AJ, Bailey VA (1935) Balance of animal populations. Part- I. Proc. Soc. London 3: 551-598.
Olson RS, Knoester DK, Adami C (2013) Critical interplay between density-dependent predation and evolution of the selfish herd. Proc Gen Evol Comp Conf (GECCO), pp 1-8.

Omkar, Pervez A (2004) Functional and numerical responses of Propylea dissecta (Mulsant) (Col., Coccinellidae). J Appl Ent 128: 140-146.

Omkar, Pervez A (2011) Functional Response of two aphidophagous ladybirds searching in tandem. Biocont Sci Tech 21: 101-111.

Pal D, Mahapatra GS (2014) A bioeconomic modeling of two-prey and one-predator fishery model with optimal harvesting policy through hybridization approach. Appl Math Comp 242: 748-763.

Pei Y, Chen L, Zhang Q, Li C (2005) Extinction and permanence of one-prey multi-predators of Holling type II function response system with impulsive biological control. J Theor Biol 235: 495-503.

Pervez A, Omkar (2003) Predation potential and handling time estimates of a generalist aphidophagous ladybird, Propylea dissecta. Biol Memoirs 29: 91-97.

Rayungsari M, Kusumawinahyu WM, Marsudi (2014) Dynamical analysis of predator-prey model with ratio-dependent functional response. Appl Math Sci 8: 1401-1410.

Sharma S, Samanta GP (2014) Dynamical behaviour of a two prey and one predator system. Differ Equa Dyn Syst 22: 2125-145.

Shuwen Z, Lingzhen D, Lansun C (2005) The study of predatorprey with defensive ability of prey and impulsive perturbations on the predator. Chaos, Solitons and Fractals 23: 631-643.

Sokol W, Howell JA (1987) The kinetics of phenol oxidation by washed cells. Biotech Bioeng 30: 921-927.

Song X, Xiang Z (2006) The prey-dependent consumption two prey one- predator models with stage structure for the predator and impulsive effects. J Theor Biol 242: 683-698.

Sunaryo MSW, Salleh Z, Mamat M (2013) Mathematical model of three species food chain with Holling type-III functional response. Int J Pure Appl Math 89: 647-657.

Tanner JT (1975) The stability and intrinsic growth rate of prey and predator populations. Ecol 56: 855-867.

Upadhyay RK, Raw SN (2011) Complex dynamics of a three species food-chain model with Holling type IV functional response. Nonlinear Analysis: Modelling and Cont 16: 353-374.

Upadhyay RK, Banerjee M, Parshad RD, Raw SN (2011) Deterministic chaos versus stochastic oscillation in a prey-predator-top predator model. Math Model Anal 16: 343-364.

Venkatesan A, Parthasarathy S, Lakshmanan M (2003) Occurrence of multiple period-doubling bifurcation route to chaos in periodically pulsed chaotic dynamical systems. Chaos, Solitons and Fractals18: 891-898.

Volterra V (1926) Variazioni e uttuazioni del numero di individui in specie animali conviventi. Mem Accad Linc 2: 31-113.

Xianning L, Lansun C (2003) Complex dynamics of Holling type II Lokta-Voltrra predator-prey system with impulsive perturbations on the predator. Chaos, Solitons and Fractals 16:311-320.

Yang J, Tang S (2009) Effects of population dispersal and impulsive control tactics on pest management. Nonlinear Anal Hybrid Syst 3: 487-500.

Zhang S, Chen L (2005) A Holling II functional response food chain model with impulsive perturbations. Chaos, Solitons and Fractals 24: 1269-1278. 